\title{
Impact of COVID-19 pandemic on patients and health professionals of a radiation oncology department at a Spanish tertiary hospital
}

\author{
Jesús Romero ${ }^{(1}{ }^{1}$, Raquel Benlloch ${ }^{1}$, Jorge Obeso ${ }^{1}$, Olga Engel ${ }^{1}$, Beatriz Gil ${ }^{1}$, Sofía Córdoba ${ }^{1}$, Irma Zapata ${ }^{1}$, \\ Marta López ${ }^{1}$, Francisca Portero ${ }^{2}$
}

${ }^{1}$ Servicio de Oncología Radioterápica; Hospital Universitario Puerta de Hierro, Majadahonda, Madrid, Spain

${ }^{2}$ Servicio de Microbiología, Hospital Universitario Puerta de Hierro, Majadahonda, Madrid, Spain

\begin{abstract}
Background: The sanitary emergency created by the COVID-19 pandemic forced us to take exceptional measures that affect decision-making and administration of treatments with radiotherapy. The aim of the study was to analyze the impact of the COVID-19 pandemic on patients and professionals in a radiation oncology department.

Materials and methods: We implement a plan with the objectives of maintaining radiotherapy treatment in those patients who need it and, at the same time, reducing the risk of spreading the virus to staff and patients. This plan included measures aimed at limiting the patient's stay in hospital, selecting those patients in whom radiotherapy cannot be delayed and protecting against infection through the use of physical protective measures.

Results: Between March 16 and May 31, 2020, 360 patients received radiotherapy in our department. In 14 patients (4.7\%) the start of treatment was delayed by an average of 28 days. Four patients had a positive COVID-19 polymerase chain reaction (PCR) $(6.6 \%$ and $1.1 \%$ of tested and all patients, respectively). Among the professionals, two PCRs were positive ( $16.6 \%$ and $4 \%$ of tested and all individuals, respectively). In the serology analysis 4 out of 50 department members were lgG positive (8\%).

Conclusions: Despite the fact that our department is located in a region with a high incidence of COVID-19 infection, the impact of the pandemic on our patients and staff has been moderate. The implementation of measures against infection and an adequate selection of patients for treatment allows radiation oncology departments to maintain clinical activity.

Key words: coronavirus; COVID-19; radiotherapy; cancer

Rep Pract Oncol Radiother 2021;26(2):237-241
\end{abstract}

\section{Introduction}

A novel coronavirus called severe acute respiratory syndrome coronavirus 2 (SARS-CoV-2) was identified in December 2019 in Wuhan, China, as the cause of a respiratory infection called coronavirus disease (COVID-19). The infection spread throughout the rest of the world and was declared a pandemic by the World Health Organization on March 11, 2020. By August 1, more than 17 million people had been infected and more than 680,000 people had died from COVID-19 according to the World Health Organization.

The disease is characterized by fever and respiratory symptoms. In most cases, patients present with mild illness, but up to $15 \%$ of patients require

Address for correspondence: Jesús Romero, Servicio de Oncología Radioterápica; Hospital Universitario Puerta de Hierro, Calle Joaquín Rodrigo, 1; Majadahonda, 28222 Madrid, Spain; e-mail: jesus.romero@salud.madrid.org

This article is available in open access under Creative Common Attribution-Non-Commercial-No Derivatives 4.0 International (CC BY-NC-ND 4.0) license, allowing to download articles and share them with others as long as they credit the authors and the publisher, but without permission to change them in any way or use them commercially 
hospitalization and oxygen supplementation, and $5 \%$ progress to severe illness with respiratory failure and multi-organ dysfunction [1].

The management of cancer patients is a challenge because they have a higher risk of contracting COVID-19 as well as developing a more serious disease because their immunosuppressed state caused by both the tumor and cancer treatments $[2,3]$. Cancer patients undergoing chemotherapy or surgery have a higher risk of clinically severe events (odds ratio: 5.34, 95\% CI: 1.80-16.18; $\mathrm{p}=0.0026)$ after adjusting for other risk factors [2]. Oncologists face the dilemma of treating patients to prevent cancer progression or, conversely, delaying cancer treatment to prevent the spread of COVID-19.

In this report we share our experience in the management of patients undergoing radiotherapy during the pandemic in a region with a high prevalence of COVID-19 infection. We analyze the impact of the COVID-19 pandemic on the delivery of treatments in a radiation oncology department and determine the effectiveness of a contingency plan in reducing the risk of contagion for patients and healthcare personnel.

\section{Materials and methods}

The university hospital Puerta de Hierro is a public tertiary hospital that covers an area with population of 462,000. Around 1,600 new patients per year are treated in the department of Radiation Oncology, which has four linear accelerators and a high-rate brachytherapy unit. The department is comprised of 50 members: 20 doctors (12 staff radiation oncologists and 8 residents), 22 radiotherapy technicians, 5 oncology nurses, 2 nursing assistants and 1 administrative secretary. Before the pandemic, approximately 30 new patients and 300 follow-up visits per week were seen, and a mean of 130 patients received radiotherapy in our department daily.

\section{Laboratory tests}

The tests were performed in the Microbiology laboratory of the hospital. The viral RNA in the nasopharyngeal swab was detected by PCR (PCR, cobas ${ }^{\circledast}$ SARS-CoV-2, Roche). COVID-19 ELISA $\operatorname{IgM}+\operatorname{IgA}$ and $\operatorname{IgG}$ (Vircell) was used for detection of specific antibodies in a blood sample.

\section{Contingency plan}

The sanitary emergency created by the pandemic forced us to take exceptional measures that affect decision-making and administration of treatments with radiotherapy or chemoradiotherapy. The two objectives of the plan were to guarantee treatment with radiotherapy in those patients who need it and to reduce the risk of spreading the virus to healthcare personnel and patients.

To meet these objectives, measures were implemented aimed at limiting the patient's stay in hospital, selecting those patients in whom radiotherapy cannot be delayed and protecting against infection through the use of physical protective measures.

Some of the measures adopted were the following:

- follow-up visits were conducted distantly by phone when appropriate;

- the first visits for new patients were maintained except in tumors in which the delay in treatment was acceptable, such as benign tumors, breast and prostate cancer;

- various hypofractionation schemes were adopted whenever possible. Some of the schedules used in the most frequent tumors were the following:

- breast - patients $>50$ years old, without boost and no chemotherapy: 5 weekly fractions of 2.66 Gy/f over 5 weeks. For the rest of patients: 15 fractions of $2.66 \mathrm{~Gy} / \mathrm{f}$ with integrated boost, if appropriate, at $3 \mathrm{~Gy} / \mathrm{f}$,

- prostate -20 fractions of $3 \mathrm{~Gy} / \mathrm{f}$ over 4 weeks,

- lung - 20 fractions of $2.66 \mathrm{~Gy} / \mathrm{f}$ over 4 weeks for non-small cell. For small cell limited stage, 15 fractions of $2.66 \mathrm{~Gy} / \mathrm{f}$ and prophylactic cranial irradiation (PCI) with 10 fractions of $2.5 \mathrm{~Gy} / \mathrm{fx}$. For extended stage in selected patients, $5 \mathrm{fx}$ of 4 Gy/f without PCI.

In patients with head and neck, cervical and lung cancers, enlargements of the overall treatment time were compensated by adding $0.6 \mathrm{~Gy}$ to the total dose for each day lost up to a maximum of $6 \mathrm{~Gy}$;

- to assist in decision making, patients were classified according to their survival expectations and the potential relief of disabling symptoms (Tab. 1);

- patients were frequently asked about the presence of fever, cough, or dyspnea, and also about potential contacts with COVID-19 patients. In suspected cases, a PCR test, chest X-ray, and laboratory tests were performed to rule out $\mathrm{CO}$ - 
Table 1. Classification of patients according to their survival expectations, risk of relapse and potential relief of disabling symptoms

\begin{tabular}{|l|l|} 
High priority & $\begin{array}{l}\text { Curative treatments with survival }>50 \% \text { in rapidly growing tumors in which delayed treatment can compromise } \\
\text { survival } \\
\text { Patients with spinal cord compression in whom radiation therapy can produce neurological recovery }\end{array}$ \\
\hline Medium priority & $\begin{array}{l}\text { Curative treatments with survival between } 10-50 \% \\
\text { Postoperative radiotherapy in patients with macroscopic residue of fast growing tumors or with 5-year recurrence } \\
\text { risk }>20 \%\end{array}$ \\
\hline $\begin{array}{l}\text { Palliative radiotherapy that produces symptomatic relief } \\
\text { Postoperative radiation therapy with complete resection and 5-year recurrence risk }<20 \% . \\
\text { Radical radiotherapy in patients in whom the delay does not impact control or those in whom hormonal therapy or } \\
\text { other treatment allows delay of treatment }\end{array}$ \\
\hline
\end{tabular}

VID-19 infection. In patients with a diagnosis of COVID-19 pneumonia and/or positive PCR, treatment was interrupted until the patient had a clinical situation that allowed the restart of radiotherapy;

- protective measures were established for all health personnel consisting of the use of surgical masks, gloves, and protective face shields. The accelerator table and all devices in the treatment room were carefully cleaned with hydroalcoholic solution before each treatment. The patients were given a surgical mask and we tried to avoid crowding in the waiting room.

\section{Results}

Between March 16 and May 31, 2020, 360 patients received radiotherapy in our department. Among them 280 patients started treatment during the period analyzed, which represents an average of 5.86 treatment initiations per day. An average of 86.65 daily radiotherapy sessions were administered in the four radiotherapy units and a total of 37 high-rate brachytherapy procedures were performed. During this period there was a $27 \%$ decrease in the number of patients treated with respect to the same period of the previous year. This decrease is attributable to a lower referral of patients for postoperative radiotherapy due to a drastic decrease in surgical interventions, as well as the delay in the start of radiotherapy in some patients.

The start of radiotherapy was delayed in 14 patients (4.7\%) according to the contingency plan or by patient decision. The mean delay time until the start of radiotherapy was 28 days (7-58).

One or more PCR tests were performed on 60 patients (Tab. 3). The test was positive only in 4 patients $(6.6 \%$ of the patients tested and $1.1 \%$ of the total of 360 patients receiving radiotherapy). The mean time for PCR negativization was 6 days. In 2 additional patients, the diagnosis of COVID-19 was established by a compatible clinical picture without performing PCR. Three patients presenting with viral pneumonia required admission and there were no deaths for COVID-19 in our series. In patients with a diagnosis of COVID-19, the mean time of interruption of radiotherapy was 20 days (6-45) and this enlargement could only be partially compensated in some patients.

One or more PCRs were performed in 12 staff members with symptoms compatible with COVID-19 infection, of which 2 were positive (16.6\% of tested individuals and $4 \%$ of all department members). In the seroprevalence study carried out in May 2020 to all members of the department, 4 out of 50 individuals (8\%) had IgG antibodies against COVID-19 (Tab. 2).

As the health crisis progressed, it was necessary to reorganize the department's units due to a 30\% decrease in the workforce motivated by the recruitment of doctors by the hospital administration to treat patients with COVID-19 and by sick leaves due to COVID-19 infection or quarantine.

\section{Discussion}

With 1063 confirmed cases and 130 deaths per 100,000 populations by May 19, 2020, the autonomous region of Madrid is one of the European areas with the highest incidence of COVID-19 infection. The pandemic has nearly collapsed the regional health system due to a low availability of diagnostic tests and protection equipment, insufficient ICU beds and mechanical ventilators, and a high num- 
Table 2. COVID-19 PCR and COVID-19 ELISA IgM and IgG results in patients and healthcare professionals

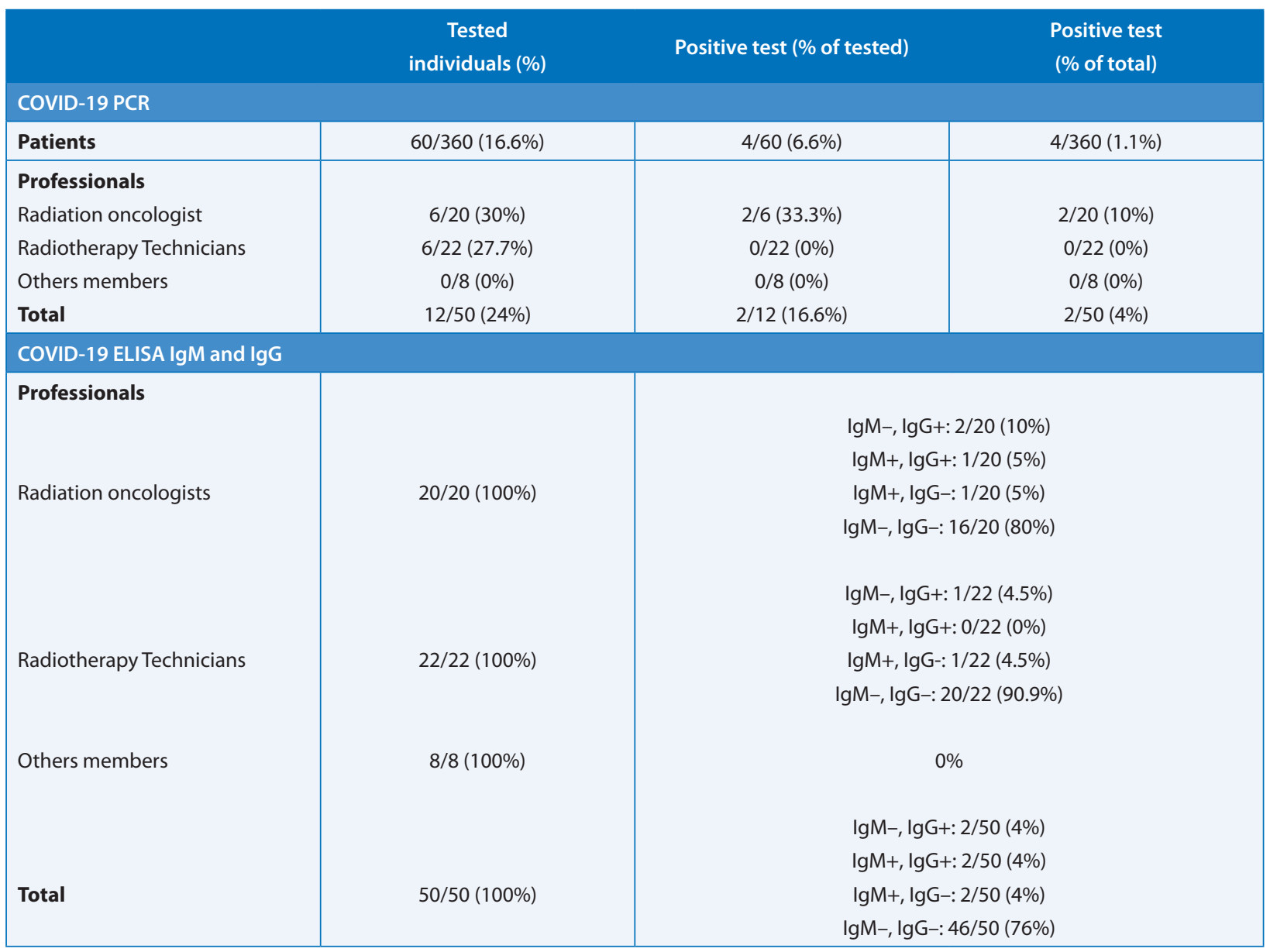

ber of healthcare professionals infected by COVID-19. To deal with the situation created by the COVID-19 pandemic we launched a contingency plan with the aim of maintaining radiotherapy treatment but reducing the spread of the virus through protective measures.

Since the beginning of the pandemic, various recommendations have been published regarding the management of radiation oncology departments [4-6]. As in our contingency plan, these recommendations are based on the limitation of the patient's stay in the hospital; use of hypofractionation schemes; selection of high-risk patients in whom treatment should be performed; and in the adoption of protection measures against $\mathrm{CO}$ VID-19 of patients and staff. However, the real impact of the COVID-19 pandemic in radiation oncology departments in terms of the number of patients and delays in radiation treatments, as well as the number of professionals and patients with COVID-19 infection has been rarely reported. Ma- licki et al. [7] analyzed the impact of precautionary measures in response to COVID-19 in a large Radiation Oncology center in Poland and found a reduction of $37 \%$ in the number of patients treated daily, which is similar to the $27 \%$ observed in our series. These authors [7] found an incidence of 2 out of 159 tested staff members with positive PCR (1.2\%) compared to $16.6 \%$ in our department. This difference is probably due to the fact that in our center PCR was only performed in individuals with symptoms or risky contact, as well as the higher incidence of COVID-19 infection in our region.

\section{Conclusions}

Despite the fact that our department is located in a region with a high incidence of COVID-19 infection, the impact of the pandemic on our patients and staff has been moderate. The implementation of measures against infection and an adequate selection of patients for treatment allow 
radiation oncology departments to maintain clinical activity

\section{Conflicts of interest}

All authors declare that they have no conflicts of interest for this work.

\section{Funding}

None declared.

\section{References}

1. Wu Z, McGoogan JM. Characteristics of and Important Lessons From the Coronavirus Disease 2019 (COVID-19) Outbreak in China: Summary of a Report of 72314 Cases From the Chinese Center for Disease Control and Prevention. JAMA. 2020; 323(13): 1239-1242, doi: 10.1001/jama.2020.2648, indexed in Pubmed: 32091533.

2. Liang W, Guan W, Chen R, et al. Cancer patients in SARS-CoV-2 infection: a nationwide analysis in China. Lancet Oncol. 2020; 21(3): 335-337, doi: 10.1016/S14702045(20)30096-6, indexed in Pubmed: 32066541.
3. Yu J, Ouyang W, Chua MLK, et al. SARS-CoV-2 Transmission in Patients With Cancer at a Tertiary Care Hospital in Wuhan, China. JAMA Oncol. 2020; 6(7): 1108-1110, doi: 10.1001/jamaoncol.2020.0980, indexed in Pubmed: 32211820.

4. Filippi AR, Russi E, Magrini SM, et al. Letter from Italy: First practical indications for radiation therapy departments during COVID-19 outbreak. Int J Radiat Oncol Biol Phys. 2020; 107(3): 597-599, doi: 10.1016/j.jijrobp.2020.03.007, indexed in Pubmed: 32199941.

5. Zhang Li, Zheng Z, Hu G, et al. Prevention and control measure to avoid cross infection during radiotherapy in coronavirus disease 2019 (COVID-19) epidemic in Wuhan, China. Radiother Oncol. 2020; 149: 104-106, doi: 10.1016/j. radonc.2020.04.011, indexed in Pubmed: 32342880.

6. Rivera A, Ohri N, Thomas E, et al. The Impact of COVID-19 on Radiation Oncology Clinics and Patients With Cancer in the United States. Adv Radiat Oncol. 2020; 5(4): 538-543, doi: 10.1016/j.adro.2020.03.006, indexed in Pubmed: 32292841.

7. Malicki J, Martenka P, Dyzmann-Sroka A, et al. Impact of COVID-19 on the performance of a radiation oncology department at a major comprehensive cancer centre in Poland during the first ten weeks of the epidemic. Rep Pract Oncol Radiother. 2020; 25(5): 820-827, doi: 10.1016/j. rpor.2020.08.001, indexed in Pubmed: 32837336. 